\title{
Identification and analysis of proton-translocating pyrophosphatases in the methanogenic archaeon Methanosarcina mazei
}

\author{
SEBASTIAN BÄUMER, ${ }^{1}$ SABINE LENTES, ${ }^{1}$ GERHARD GOTTSCHALK ${ }^{1,2}$ and UWE \\ DEPPENMEIER ${ }^{1,3}$ \\ 1 Abteilung Allgemeine Mikrobiologie, Institut für Mikrobiologie und Genetik, Georg-August-Universität, Grisebachstr. 8, 37077 Göttingen, Germany \\ ${ }^{2}$ Göttingen Genomics Laboratory, Institut für Mikrobiologie und Genetik, Georg-August-Universität, Grisebachstr. 8, 37077 Göttingen, Germany \\ 3 Author to whom correspondence should be addressed (udeppen@gwdg.de)
}

Received June 12, 2001; accepted August 14, 2001; published online September 21, 2001

Summary Analysis of genome sequence data from the methanogenic archaeon Methanosarcina mazei Gö1 revealed the existence of two open reading frames encoding protontranslocating pyrophosphatases (PPases). These open reading frames are linked by a 750-bp intergenic region containing TC-rich stretches and are transcribed in opposite directions. The corresponding polypeptides are referred to as Mvp1 and Mvp2 and consist of 671 and 676 amino acids, respectively. Both enzymes represent extremely hydrophobic, integral membrane proteins with 15 predicted transmembrane segments and an overall amino acid sequence similarity of 50.1\%. Multiple sequence alignments revealed that Mvp 1 is closely related to eukaryotic PPases, whereas Mvp2 shows highest homologies to bacterial PPases. Northern blot experiments with RNA from methanol-grown cells harvested in the mid-log growth phase indicated that only Mvp2 was produced under these conditions. Analysis of washed membranes showed that Mvp2 had a specific activity of $0.34 \mathrm{U} \mathrm{mg}$ (protein) ${ }^{-1}$. Proton translocation experiments with inverted membrane vesicles prepared from methanol-grown cells showed that hydrolysis of $1 \mathrm{~mol}$ of pyrophosphate was coupled to the translocation of about $1 \mathrm{~mol}$ of protons across the cytoplasmic membrane. Appropriate conditions for $m v p 1$ expression could not be determined yet. The pyrophosphatases of M. mazei Gö1 represent the first examples of this enzyme class in methanogenic archaea and may be part of their energy-conserving system.

Keywords: energy conservation, inorganic pyrophosphate, methanogenesis, proton pump, pyrophosphatase.

Abbreviations: DCCD, $N, N^{\prime}$-dicyclohexylcarbodiimide; PPase, inorganic pyrophosphatase; $\mathrm{PPi}$, inorganic pyrophosphate; $\Delta p$, proton motive force.

\section{Introduction}

Inorganic pyrophosphate (PPi) is formed in several enzymatic reactions of various metabolic pathways (e.g., deoxyribonucleic acid and ribonucleic acid polymerization and amino acid and fatty acid activation). It is supposed that subsequent hy- drolysis of PPi by pyrophosphatases shifts the overall reaction equilibrium toward product formation. However, according to Rea and Sanders (1987), this assumption may be too restrictive because a considerable amount of metabolic energy would be lost as heat. Alternatively, it is possible that pyrophosphatases utilize a portion of the PPi anhydride bond energy for the generation of a transmembrane proton gradient that could then be used to drive endergonic reactions.

Two families of pyrophosphatases have been identified in the three domains of life. The first class encompasses a wide variety of soluble cytoplasmic enzymes that are not involved in energy conservation (Silvula et al. 1999). The second class comprises tightly membrane-bound pyrophosphatases that were first isolated from vacuolar membranes of higher plants and algae (Maeshima 2000). These enzymes translocate protons across the vacuolar membrane, thereby maintaining the acidic vacuolar interior milieu. Proton-translocating pyrophosphatases have also been found in the cytoplasmic membrane of several bacteria such as Rhodospirillum rubrum (Baltscheffsky et al. 1998), Thermotoga maritima (Nelson et al. 1999), Streptomyces coelicolor (Redenbach et al. 1996), Synthrophus gentianae (Schöcke and Schink 1998) and in the hyperthermophilic archaeon Pyrobaculum aerophilum (Drozdowicz et al. 1999, Drozdowicz and Rea 2001).

In this report we show that proton-translocating pyrophosphatases are also present in the methanogenic archaeon Methanosarcina mazei Gö1. This organism derives its metabolic energy from the conversion of $\mathrm{H}_{2}$ plus $\mathrm{CO}_{2}$, acetate, methanol or methylamines to methane. Redox reactions of methanogenesis are partly catalyzed by membrane-bound enzymes that generate or use electrochemical ion gradients (Deppenmeier et al. 1996). The $\mathrm{H}_{2}$ :heterodisulfide oxidoreductase and the $\mathrm{F}_{420} \mathrm{H}_{2}$ :heterodisulfide oxidoreductase are novel electron transport systems that are able to generate a proton motive force by redox-driven proton translocation (Deppenmeier et al. 1999). A membrane-bound $\mathrm{A}_{1} \mathrm{~A}_{0}$-ATP synthase utilizes the electrochemical proton gradient for ATP synthesis (Müller et al. 1999). The pyrophosphatases found in M. mazei Gö1 represent another class of proton-translocating 
enzymes in methanogens and might be involved in a new energy-conserving system.

\section{Materials and methods}

Growth of cells

Methanosarcina mazei Gö1 (DSM 3647) was grown in 1-1 glass bottles or, for mass culturing, in 20-1 carboys on $150 \mathrm{mM}$ methanol in a medium described previously (Hippe et al. 1979), supplemented with $1 \mathrm{~g}^{-1}$ sodium acetate.

\section{Preparation of washed vesicles and washed membranes}

Washed inverted vesicles of strain Gö1 were prepared as described by Ide et al. (1999), except that the final protein concentration was $10-15 \mathrm{mg} \mathrm{ml}^{-1}$. Washed membranes from M. maze $i$ were prepared according to the method of Abken and Deppenmeier (1997).

\section{Measurement of proton translocation}

Proton translocation was monitored as described previously (Ide et al. 1999). Briefly, $3 \mathrm{ml}$ of $40 \mathrm{mM} \mathrm{KSCN}$ solution containing $0.5 \mathrm{M}$ sucrose, $1 \mathrm{mg} \mathrm{ml}^{-1}$ resazurin and $10 \mathrm{mM}$ dithioerythritol was added to a nitrogen-flushed, 11-ml reaction vessel, followed by addition of 50-80 $\mu$ l of washed inverted vesicles (1-1.4 mg protein per assay). Proton uptake coupled to pyrophosphate cleavage was assessed by monitoring the $\mathrm{pH}$ of the solution with a $\mathrm{pH}$ electrode connected to a chart recorder during the hydrolysis of 5 to $40 \mathrm{nmol} \mathrm{Na}$ pyrophosphate (20 mM aqueous stock solution). The uncoupler 3,5-di-tertbutyl-4-hydroxybenzylidenemalononitrile was added to a final concentration of $12 \mathrm{nmol} \mathrm{mg}{ }^{-1}$ membrane protein where indicated (Figure 4). Changes in $\mathrm{pH}$ were calibrated with standard solutions of $\mathrm{HCl}$ or $\mathrm{NaOH}$.

\section{Determination of the cytoplasmic phosphate concentration}

Five-ml cultures of M. mazei were harvested in the exponential growth phase and washed three times with $25 \mathrm{mM}$ MOPS plus $0.5 \mathrm{M}$ sucrose to remove phosphate contaminants. After cell sonication, protein was precipitated by heating at $100{ }^{\circ} \mathrm{C}$ for 5 min and removed by centrifugation. The supernatant was used for determination of the cytoplasmic phosphate concentration.

\section{Enzyme activity assays}

Pyrophosphatase activity was determined in 1.8-ml glass vessels containing $25 \mathrm{mM}$ MOPS buffer $\mathrm{pH} 7$ and washed membranes (about $1 \mathrm{mg}$ membrane protein). Sodium pyrophosphate and $\mathrm{MgCl}_{2}$ were added from $0.1 \mathrm{M}$ aqueous stock solutions to final concentrations of 4 and $3 \mathrm{mM}$, respectively. Membranes were preincubated with $\mathrm{MgCl}_{2}$ at $37{ }^{\circ} \mathrm{C}$ and the reaction was started by the addition of pyrophosphate. To examine PPi hydrolysis, 50- $\mu$ l samples were withdrawn at intervals and added to $10 \mu \mathrm{l}$ concentrated $\mathrm{HClO}_{4}$ to stop enzyme activity. Precipitated protein was removed by centrifugation. The release of free phosphate due to pyrophosphate hydrolysis was determined as described by Saheki et al. (1985) with the following specifications. Each $25-\mu$ sample prepared as de- scribed above was added to $750 \mu \mathrm{l}$ of a $15 \mathrm{mM}$ ammonium molybdate solution with $70 \mathrm{mM}$ zinc acetate $(\mathrm{pH} 5.0 \mathrm{HCl})$ and reduced with $250 \mu$ freshly prepared $10 \%$ sorbic acid solution $(\mathrm{pH} 5.0 \mathrm{NaOH})$. After incubation for $20 \mathrm{~min}$, absorbance of samples at $850 \mathrm{~nm}$ was measured against a phosphate blank sample. Concentrations were calculated from a standard curve of $0-250 \mu \mathrm{M} \mathrm{KH}_{2} \mathrm{PO}_{4}$.

\section{Construction of ${ }^{32} P$-labeled probes and northern blot analysis}

For the construction of specific DNA probes for studies of pyrophosphatase gene expression in M. mazei Gö1, non-homologous sequence regions of $m v p 1$ (Probe 1: bp 743-1089) and $m v p 2$ (Probe 2: bp 710-1035) were amplified by polymerase chain reaction (PCR) using chromosomal DNA as template. The DNA fragments were isolated from a $0.8 \%$ agarose gel and purified with a gel extraction kit (Qiaex II, Qiagen, Hilden, Germany). Probes were radiolabeled with $\left[\alpha-{ }^{32} \mathrm{P}\right] \mathrm{dATP}$ (Hartmann, Braunschweig, Germany) with a random primer labeling kit (Boehringer, Mannheim, Germany), and purified by gel chromatography (NAP 10, Pharmacia, Uppsala, Sweden). Total cellular RNA was prepared from methanol-grown M. mazei Gö1 (harvested in the mid-log phase) with an RNA isolation kit (RNeasy, Qiagen) according to the manufacturer's instructions. Northern blot analysis was performed as described previously (Deppenmeier et al. 1995).

\section{GenBank accession numbers}

The nucleotide sequences and corresponding amino acid sequences for genes encoding proton-translocating and soluble PPases from M. maze $i$ have been deposited in the GenBank database under Accession Numbers AF312701 ( $m v p 1$ and $m v p 2$ ) and AF312700 (gene encoding a soluble PPase).

\section{Results}

\section{Molecular biological analysis}

DNA sequence data from the $M$. mazei Gö1 genome project (Göttingen Genomics Laboratory, Göttingen, Germany) revealed two open reading frames encoding hypothetical plant V-type pyrophosphatase homologs. The genes were designated mvp 1 and mvp2 (Methanosarcina vacuolar-type pyrophosphatase). They are linked by a 750-bp intergenic region containing TC-rich stretches and are transcribed in opposite directions (Figure 1). Typical archaeal promoter sequences of the TATA-box initiator type (Brown et al. 1989) were found upstream of both genes. Bacterial-type ribosomal binding sites immediately preceded the genes.

The deduced polypeptides of $m v p 1$ (N-terminus MERLIFT) and $m v p 2$ (N-terminus MDMLIY) consist of 671 (69.1 kDa) and 676 amino acids $(69.2 \mathrm{kDa})$, respectively. Both enzymes represent extremely hydrophobic, integral membrane proteins with 15 predicted transmembrane segments. The overall amino acid sequence similarity between Mvp1 and Mvp2 was 50.1\%. The deduced amino acid sequences of $m v p 1$ and $m v p 2$ were compared with proton-translocating PPases from other organ- 


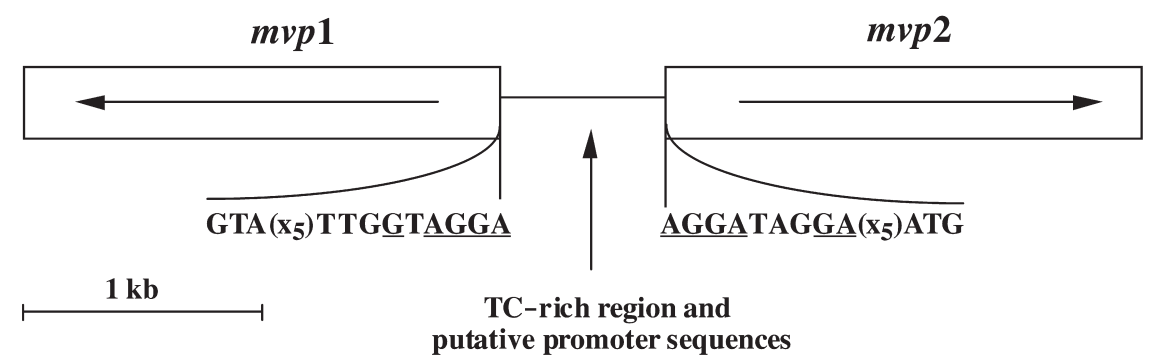

Figure 1. Organization of genes encoding proton-translocating pyrophosphatases in M. mazei Gö1. Base pairs contributing to the ribosomal binding site (RBS) are underlined $\left(\mathrm{A} / \mathrm{GGG}_{\mathrm{G}}^{\mathrm{A}} / \mathrm{GGGTG}^{\mathrm{A}} / \mathrm{G}=\right.$ consensus sequence of the RBS in M. mazei); black arrows indicate the direction of transcription of $m v p 1$ and $m v p 2$.

isms. Alignments indicated sequence similarities in the range of 35.5 to $50.6 \%$. For Mvp1, the highest scores were obtained for PPases of the eukaryotes Acetabularia mediterrana (50.6\%), Nicotiana tabacum $(45.1 \%)$ and Chara corallina (45.4\%). In contrast, Mvp2 showed the highest sequence similarities to PPases from the bacterium Rhodospirillium rubrum (45.6\%) and the archaeon Pyrobaculum aerophilum (42.9\%).

\section{Analysis of pyrophosphatase gene expression}

For analysis of the expression pattern of $m v p 1$ and $m v p 2$, specific DNA probes for both genes were synthesized by PCR using chromosomal DNA as template. Total RNA isolated from methanol-grown cells was used for Northern blot analysis. One major band with a size of $1.8 \mathrm{~kb}$ was obtained when RNA from these cells was hybridized with the $m v p 2$-specific probe (Figure 2). No specific signals were found with the $m v p 1$-specific probe. The weak signals at 2.5 and $1.4 \mathrm{~kb}$ are probably caused by nonspecific binding to $23 \mathrm{~S}$ rRNA and $16 \mathrm{~S}$ rRNA, respectively (Deppenmeier et al. 1995). Because the $m v p 2$ transcript has a length of $1.8 \mathrm{~kb}$, it is evident that only this gene, and not mvp1, is expressed by $M$. mazei cells grown on

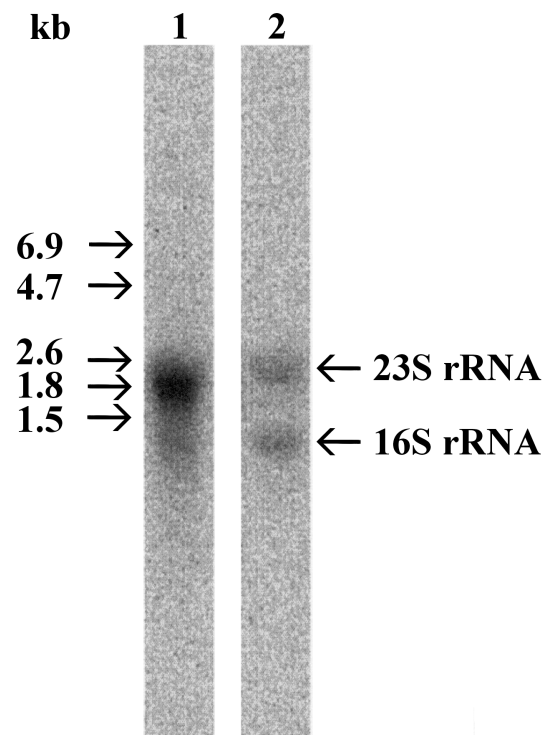

Figure 2. Analysis of expression of $m v p 1$ and $m v p 2$ from M. mazei Gö1. Northern blotting was performed using total RNA from methanol-grown cells. Each lane contained $5 \mu \mathrm{g}$ RNA. The blots were hybridized with probes specific for $m v p 2$ (Lane 1) and $m v p 1$ (Lane 2). methanol. Because the following experiments were performed with methanol-grown cells harvested in the mid-log phase, pyrophosphatase activity was attributable to Mvp2 exclusively and there was no interference from Mvp1.

\section{Biochemical analysis}

To determine the cellular localization of Mvp2, cell lysates of M. mazei $\mathrm{Gö1}$ were centrifuged at $120,000 \mathrm{~g}$ for $2 \mathrm{~h}$ to separate the membraneous fraction from the cytoplasm. Almost $100 \%$ of the PPase activity was present in the pellet. The pellet was resuspended and centrifuged at 100,000 $g$ for $2 \mathrm{~h}$. As before, almost $100 \%$ of the PPase activity was detected in the membranous fraction, indicating that the enzyme is located in the cytoplasmic membrane. The activity of the membrane-bound PPase was linearly dependent on the concentration of membrane protein up to $1 \mathrm{mg} \mathrm{ml}^{-1}$ (data not shown). Furthermore, the enzyme was air-stable for several hours. The protein showed maximal activity $\left(0.34 \mu \mathrm{mol} P P i\right.$ hydrolyzed $\mathrm{min}^{-1} \mathrm{mg}$

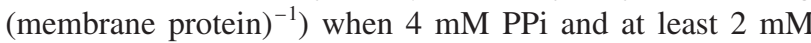
$\mathrm{Mg}^{2+}$ were added to the reaction mixture (Figure 3). Lower concentrations of these ions resulted in a decrease in the reaction rate. As already observed for other PPases (Maeshima and Yoshida 1989), the Mvp2 protein was inhibited by $N, N^{\prime}$-dicyclohexylcarbodiimide (DCCD), indicated by $\mathrm{IC}_{50}$ values of 1.5 $\mu$ mol DCCD per mg membrane protein (not shown).

In addition to $m v p 1$ and $m v p 2$, genome sequencing of M. mazei revealed the presence of a gene encoding a soluble pyrophosphatase (GenBank Accession No. AF312700). However, the cytoplasmic fraction did not contain PPase activity. Thus, it appears that the soluble PPi-hydrolyzing enzyme was not produced when cells were grown on methanol and harvested in the mid-log phase.

Multi-alignments and biochemical analysis indicated that Mvp2 is a membrane-bound proton-translocating pyrophosphatase. To verify this hypothesis, washed inverted vesicles from $M$. mazei Gö1 were tested for their ability to couple PPi hydrolysis to the transfer of protons across the cytoplasmic membrane. Concentrated vesicles were diluted with a sucrose/thiocyanate solution under an atmosphere of molecular nitrogen and pulsed with PPi as shown in Figure 4. When the substrate was added, there was a short period of alkalinization, as a result of rapid proton movement into the lumen of the inverted vesicles. In the second phase, reacidification was observed until a stable $\mathrm{pH}$ value was reached. It is thought that this reacidification was caused by decay of the generated 


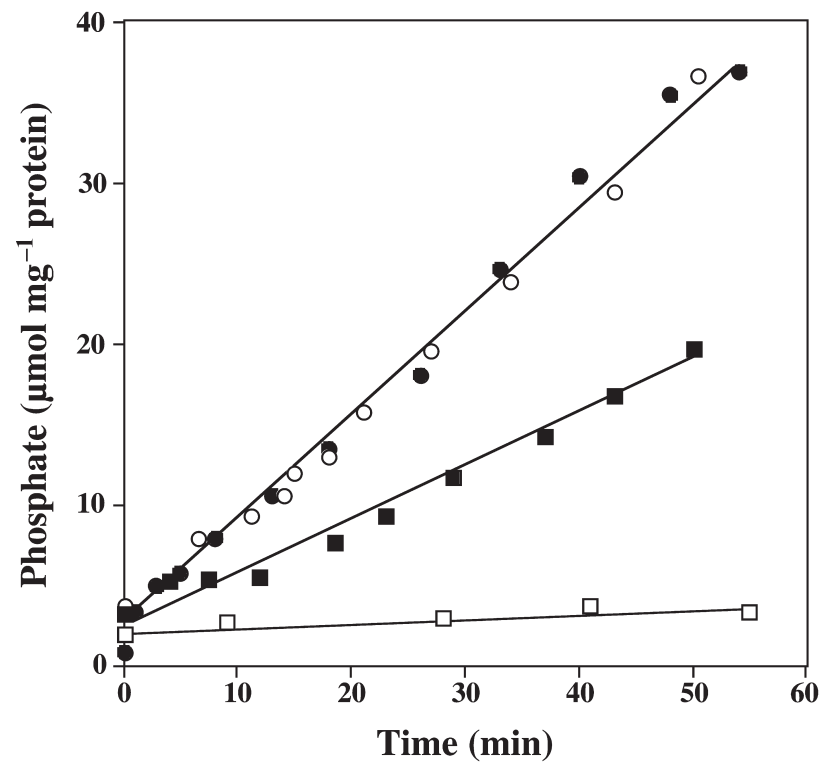

Figure 3. Magnesium ion dependence of phosphate formation from PPi hydrolysis as catalyzed by washed cytoplasmic membranes from M. mazei Gö1. The amount of PPi hydrolyzed was determined by analysis of the phosphate concentration in the reaction mixture as described in Materials and methods. Symbols: no addition of $\mathrm{Mg}^{2+}(\square)$; addition of $\mathrm{Mg}^{2+}$ to a final concentration of $1 \mathrm{mM}(\boldsymbol{\square}), 2 \mathrm{mM}(\bigcirc)$ and $3 \mathrm{mM}(\bullet)$.

chemical potential $\left(\Delta \mu_{\mathrm{H}^{+}}\right)$by passive diffusion of protons from vesicle lumena back to the medium. The addition of the protonophore SF 6847 led to complete inhibition of reversible alkalinization (Figure 4), indicating that protons function as coupling ions. After calibration of the system, an average of $0.47 \pm 0.12$ protons translocated per PPi hydrolyzed was calculated from more than 30 experiments. Taking into account that about $50 \%$ of the membrane structures present in the vesicle preparations are unable to establish a proton gradient (Dep- penmeier et al. 1999), the hydrolysis of $1 \mathrm{~mol} \mathrm{PPi}$ is coupled to the translocation of about $1 \mathrm{~mol}$ of protons.

\section{Discussion}

\section{Energetic considerations}

Methanogenesis in M. mazei is not coupled to substrate-level phosphorylation. Instead, it has been shown that energy is conserved by a chemiosmotic mechanism (Deppenmeier et al. 1999). The membrane-bound electron transport system of this organism is able to translocate $4 \mathrm{~mol}$ of protons in the course of the generation of $1 \mathrm{~mol}$ of methane. Thus, it is evident that M. mazei has only a limited ability to generate electrochemical ion gradients. Keltjens et al. (1988) reported that methane formation is coupled to PPi synthesis in Methanobacterium thermoautotrophicum. However, it was later shown by Ellermann et al. (1989) that these experiments were not reproducible. Accordingly, methanogenesis from methanol plus $\mathrm{H}_{2}$ catalyzed by washed cell suspensions of $M$. mazei Gö1 was not coupled to PPi synthesis (unpublished results). Irrespective of this controversy, it is evident that several core biosynthetic pathways generate PPi (e.g., DNA and RNA synthesis, amino acid activation, polysaccharide synthesis and formation of fatty acyl-CoA (Maeshima 2000)). Thus, the idea that PPi may function as an energy-rich intermediate for ATP synthesis is reasonable. This hypothesis is supported by the finding that the PPi concentration in the cytoplasm of M. mazei Gö1 is low ( $<0.2 \mathrm{mM}$ ), even though soluble PPase activity is lacking. Thus, the membrane-bound PPases may be the only enzymes responsible for the disposal of cytosolically produced PPi, thereby pulling the biosynthetic reactions to completion. In addition, the enzymes salvage a portion of the free energy of PPi hydrolysis by proton translocation and the formation of a proton gradient. The actual free energy change for PPi hydrolysis in the cytoplasm has been calculated to be $27.3 \mathrm{~kJ} \mathrm{~mol}^{-1}$ at pH 7.3 (Davis et al. 1993). The electrochemical potential

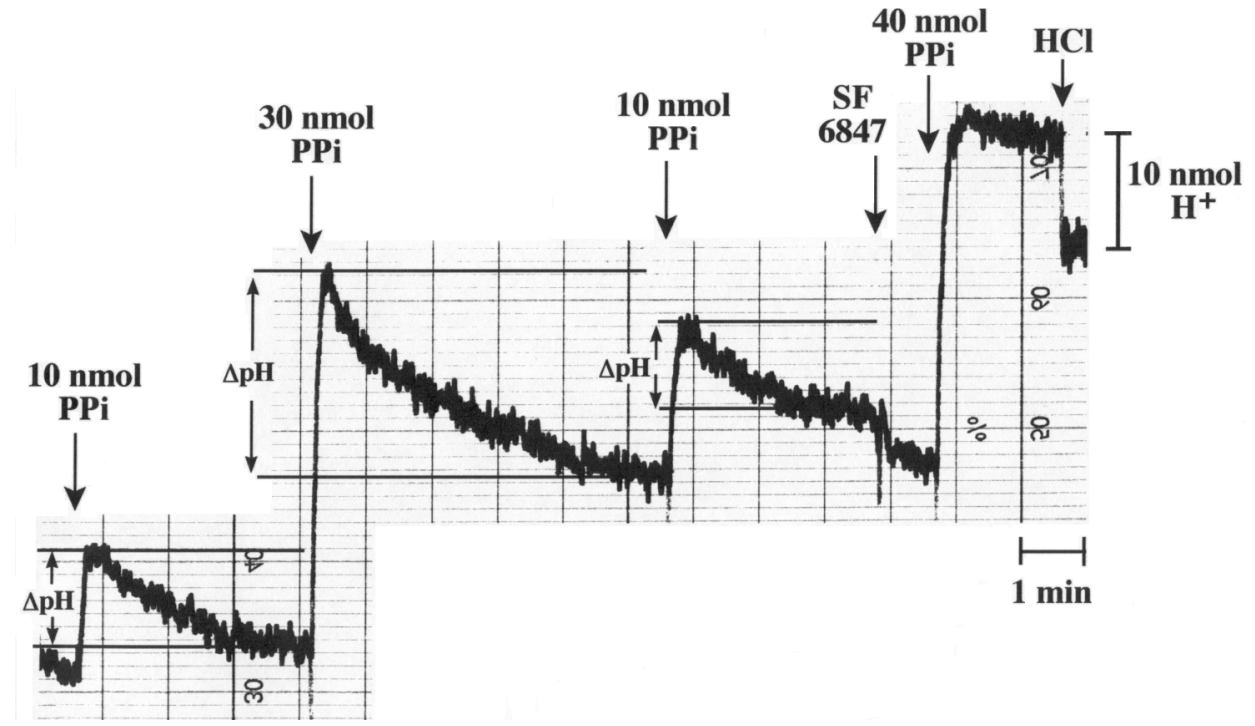

Figure 4. Proton-translocating activity of washed inverted vesicles of M. mazei Gö1 in the presence of PPi. The experiments were performed as described in Materials and methods. The amount of translocated protons was calculated from the difference between maximal alkalinization and the final baseline after reacidification as indicated for each experiment (black arrows). The difference between the starting baseline and the final baseline was due to alkalinization by the addition of PPi to the weakly buffered reaction mixture. The system was calibrated by injection of $10 \mathrm{nmol} \mathrm{HCl}$. 
that can be established by a given proton pump depends on the free energy change $(\Delta G)$ of the driving reaction and on the number of protons transported per cycle $(\Delta G=n F \Delta p$, where $n=$ number of protons, $F=$ Faraday's constant, and $\Delta p=$ proton motive force). It has been shown that $\Delta p$ of $M$. mazei is about $-150 \mathrm{mV}$ (Peinemann 1989). Thus, for the translocation of $1 \mathrm{~mol}$ of protons, a free energy change of at least $14.6 \mathrm{~kJ}$ is necessary. Taking the above-mentioned free energy change of $27.3 \mathrm{~kJ} \mathrm{~mol}^{-1}$ into account, PPi hydrolysis coupled to proton transfer is still an exergonic process that can function as a driving force for biosynthetic reactions. The advantage of a membrane-integral PPase is that some of the energy is conserved in the form of a transmembrane electrochemical proton gradient, whereas soluble PPases merely dissipate all free energy thermally.

\section{Putative enzyme structure and sequence characteristics}

Sequence comparisons and computer-assisted topological and functional analyses demonstrated that the enzyme encoded by $m v p 2$ belongs to the membrane-associated proton-translocating family of PPases. The gene mvp 1 was not expressed in methanol-grown cells, but multiple alignments of the deduced amino acid sequence showed that Mvp1 also belongs to this class of enzymes. The most thoroughly investigated proton-translocating PPases are those found in the vacuolar membranes of plant cells (Maeshima 2000) and the cytoplasmic membrane of the photosynthetic bacterium Rhodospirillum rubrum (Baltscheffsky et al. 1998). Structural studies indicate that these enzymes are composed of only one subunit with a molecular mass of 67.5 to $80.8 \mathrm{kDa}$ (Maeshima 2000). Evidence for the presence of proton-translocating PPases has also been found in several other organisms such as the thermophilic bacterium Thermotoga maritima (Nelson et al. 1999), the marine alga Acetabularia mediterranea (Ikeda et al. 1999), the parasitic protist Trypanosoma cruzi (Hill et al. 2000), the malaria parasite Plasmodium falciparum (Luo et al. 1999), the syntrophic bacterium Syntrophus gentianae (Schöcke and Schink 1998) and the soil bacterium Streptomyces coelicolor (Redenbach et al. 1996; for a review see Drozdowicz and Rea 2001). Recently, Drozdowicz et al. (1999) described a vacuolar-type membrane pyrophosphatase from the hyperthermophilic archaeon Pyrobaculum aerophilum, the first report of the presence of this category of pump in an archaeon. This discovery indicates that these proteins are distributed among all three domains of life, a conclusion supported by the discovery of PPase orthologs in the methanogenic archaeon M. mazei. Interestingly, genes encoding proton-translocating PPases are not present in the genomes of Methanobacterium thermoautotrophicum and Methanococcus jannaschii (Bult et al. 1996, Smith et al. 1997). Moreover, they are not found in the sulfate-reducing archaeon Archaeoglobus fulgidus (Klenk et al. 1997), a close relative of M. mazei.

The deduced amino acid sequences of $m v p 1$ and $m v p 2$ possess most of the structural features characteristic of PPases (Figure 5). Because of the evolutionary distance between methanogenic archaea and other organisms containing PPases, the evaluation of highly conserved amino acid sequences may contribute to the identification of sequence motifs likely involved in core catalysis by all V-type PPases.

Sixteen transmembrane helices were predicted for both Mvp1 and Mvp2 with the TopPredII program. Residues 656 to 676 at the C-terminus met the criterion for a transmembrane $\alpha$-helix. However, as pointed out by Drozdowicz et al. (1999), this structure is unlikely because protein fusions of apo-

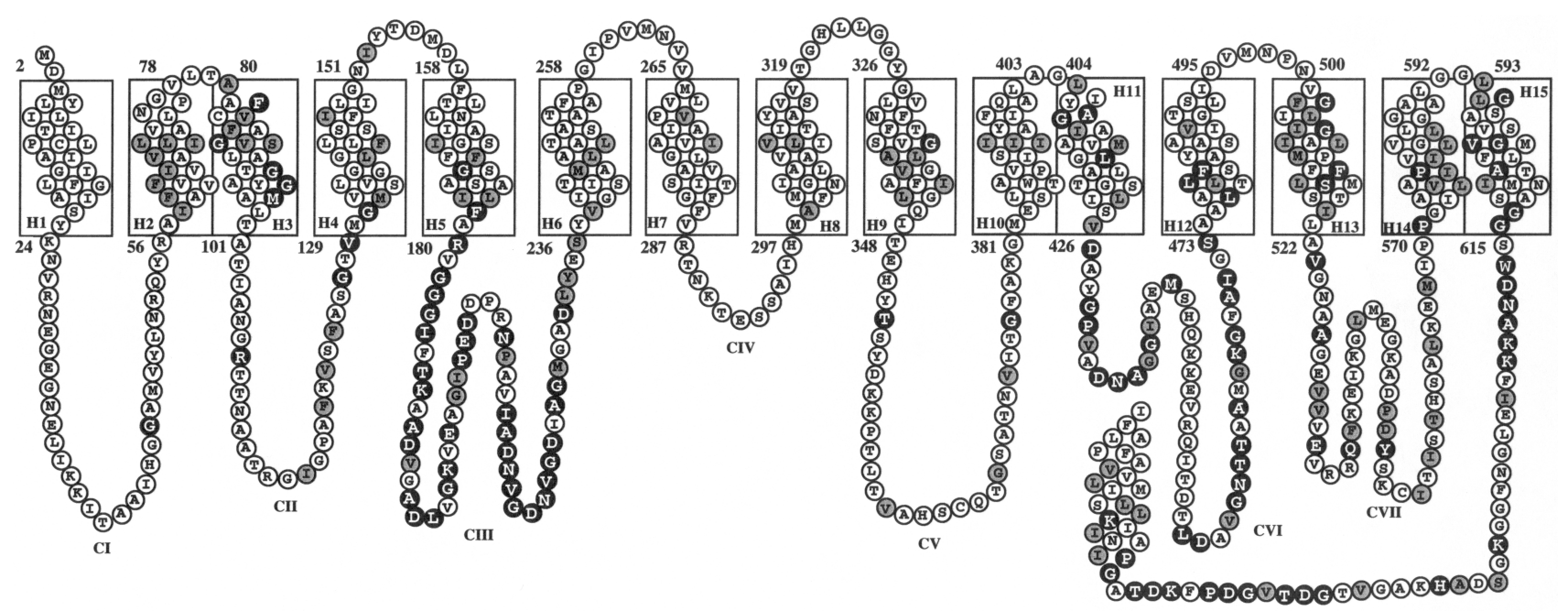

Figure 5. Predicted topology of Mvp2 and identification of conserved amino acids in the family of proton-translocating PPases. The topology prediction was performed with the program TopPredII. Multi-alignments for the identification of identical and functionally homologous amino acid residues were generated with the program MegAlign. Functionally homologous amino acids were defined as follows: acidic $=\mathrm{D}, \mathrm{E} ;$ basic $=\mathrm{H}, \mathrm{K}$, R; hydrophobic = A, F, I, L, M, P, V, W; polar = C, G, N, Q, S, T, Y. The amino acid sequence of proton-translocating PPases from the following species were compared with Mvp2: Acetabularium mediterrana, Arabidopsis thaliana, Beta vulgaris, Plasmodium falciparum, Pyrobaculum aerophilum, Rhodospirillum rubrum, Streptomyces coelicolor, Thermotoga maritima and M. mazei (Mvp1). Residues found in all sequences are shown in white on a black background. Functionally homologous amino acids are indicated in black on a grey background. 
aequorin and the $\mathrm{C}$-terminus of the vacuolar PPase in transgenic Arabidopsis plants indicated that the C-terminus of the enzyme is located in the cytoplasm. Moreover, antibody binding to the C-terminus inhibited PPi hydrolysis. Therefore, it was supposed that the $\mathrm{C}$-terminus is close to the catalytic site of the cytoplasmic loop III (Takasu et al. 1997). Considering the fundamental correspondence of the putative topology of Mvp1 and Mvp2 to that of other V-type PPases, a basic uniformity of secondary structure is most likely (Zhen et al. 1997). Therefore, the C-terminus of the methanogenic proteins may also form a helix-like structure in the cytoplasm. In summary, Mvp2 (Figure 5) is predicted to form 15 transmembrane spans that are connected by large cytoplasmic loops and relatively small outside loops. A multiple alignment of PPase sequences from Eukarya (Arabidopsis, Beta, Acetabularia and Plasmodium), Bacteria (Rhodospirillum, Streptomyces and Thermotoga) and Archaea (Pyrobaculum, M. mazei Mvp1 and Mvp2) was performed (not shown) and conserved residues are shown in white on a black background in Figure 5.

The greatest similarities between the Mvp2 sequence and the sequences of other proton-translocating PPases were detected within the hydrophilic loops III and VI with cytoplasmic orientation and the C-terminal tail, all of which are probably part of the substrate-binding and hydrolysis domain or contribute to it (Takasu et al. 1997). Moreover, there are highly conserved Gly residues in Helices 3, 4, 5, 9, 13 and 15 that could be responsible for tight localization of the corresponding helices.

Furthermore, several sequence motifs and residues assumed or demonstrated to be essential for catalysis by plant PPases are also present in the methanogenic enzymes. Comparison of all proton-translocating PPases has revealed one remarkably conserved segment (Maeshima 2000) located in the cytosolic loop III and containing the putative catalytic motif $\mathrm{Dx}_{7} \mathrm{KxE}$ found in soluble and membrane-associated PPases (Rea et al. 1992). The sequence TKAADVGADLVGKVEA from Mvp2 corresponds to this region and exactly matches the respective segment in vacuolar PPases of land plants. Is has been proposed that this subdomain participates directly in substrate and $\mathrm{Mg}^{2+}$ binding (Rea et al. 1992). Mutational analysis of the proton-translocating PPase from mung bean (Maeshima 2000, Nakanishi et al. 2001) showed that the underlined charged residues are essential for enzymatic activity (D191, K199 and E201 in Mvp2; see Figure 5).

In addition, other residues may be essential for formation of the catalytic site. Substitution of E305 and D504 in the proton-translocating PPase from Arabidopsis (Zhen et al. 1997) resulted in loss of hydrolytic activity and proton translocation (E235 and D426 in the methanogenic enzymes). The cytoplasmic loop V of many plant proton-translocating PPases contains the span-loop interface motif TEYYTS (TEHYTS in Mvp2), which encompasses a Glu residue also implicated in coupling of PPi hydrolysis to proton translocation (Zhen et al. 1997). A carbodiimide-reactive Glu residue has been identified within the C-terminal part of the enzyme from pumpkin (Rea and Poole 1993). It was proposed that the DCCD-sensi- tive residue is near the $\mathrm{Mg}^{2+}$-binding site. However, this amino acid is not conserved in the prokaryotic enzymes. Another DCCD-reactive site (D283) has been detected in the proton-translocating PPase from Vigna radiata that could be involved in the catalytic cycle (Yang et al. 1999). This residue is part of a sequence block (IADNVGDNVGD, loop CIII) that is conserved throughout all proton-translocating PPases.

In summary, it is evident that the amino acid sequences of Mvp1 and Mvp2 from the methylotrophic methanogen M. mazei contain all the residues believed to be essential for PPi hydrolysis and proton translocation. This finding was verified by biochemical experiments indicating that at least Mvp2 is able to catalyze the aforementioned reactions. Further experiments are aimed at determining conditions under which $m v p 1$ is expressed and analyzing the function of the corresponding protein in the metabolism of M. mazei.

\section{Acknowledgments}

This work was supported by a grant (De 488/2-5) of the Deutsche Forschungsgemeinschaft (Bonn-Bad Godesberg), by the DFG priority program 1070 (De 488/6-1) and by a grant of the Ministry of Science and Culture of the state Lower Saxony.

\section{References}

Abken, H.J. and U. Deppenmeier. 1997. Purification and properties of an $\mathrm{F}_{420} \mathrm{H}_{2}$ dehydrogenase from Methanosarcina mazei Gö1. FEMS Lett. 154:231-237.

Baltscheffsky, M., S. Nadanaciva and A. Schultz. 1998. A pyrophosphate synthase gene: molecular cloning and sequencing of the cDNA encoding the inorganic pyrophosphate synthase from Rhodospirillum rubrum. Biochim. Biophys. Acta 1364:301-306.

Brown, J.W., C.J. Daniels and J.N. Reeve. 1989. Gene structure, organization, and expression in archaebacteria. Crit. Rev. Microbiol. 16:287-338.

Bult, C.J., O. White, G.J. Olsen, et al. 1996. Complete genome sequence of the methanogenic archaeon Methanococcus jannaschii. Science 273:1058-1073.

Davis, J.M., R.J. Poole and D. Sanders. 1993. The computed free energy change of hydrolysis of inorganic pyrophosphate and ATP: apparent significance for inorganic-pyrophosphate-driven reactions of intermediary metabolism. Biochim. Biophys. Acta 1141: 29-36.

Deppenmeier, U., M. Blaut, S. Lentes, C. Herzberg, and G. Gottschalk. 1995. Analysis of the $v h o$ GAC and $v h t$ GAC operons from Methanosarcina mazei strain Gö1, both encoding a membranebound hydrogenase and a cytochrome b. Eur. J. Biochem. 227: 261-269.

Deppenmeier, U.,V. Müller and G. Gottschalk. 1996. Pathways of energy conservation in methanogenic Archaea. Arch. Microbiol. 165:149-163.

Deppenmeier, U., T. Lienard and G. Gottschalk. 1999. Novel reactions involved in energy conservation by methanogenic archaea. FEBS Lett. 457:291-297.

Drozdowicz, Y.M. and P.A. Rea. 2001. Vacuolar $\mathrm{H}^{+}$pyrophosphatases: from the evolutionary backwaters into the mainstream. Trends Plant Sci. 6:206-211. 
Drozdowicz, Y.M., Y.P. Lu, V. Patel, S. Fitz-Gibbon, J.H. Miller and P.A. Rea. 1999. A thermostable vacuolar-type membrane pyrophosphatase from the archaeon Pyrobaculum aerophilum: implications for the origins of pyrophosphate-energized pumps. FEBS Lett. 460:505-512.

Drozdowicz, Y.M., J.C. Kissinger and P.A. Rea. 2000. AVP2, a sequence-divergent, $\mathrm{K}^{+}$-insensitive $\mathrm{H}^{+}$-translocating inorganic pyrophosphatase from Arabidopsis. Plant Physiol. 123:353-363.

Ellermann, J., S. Rospert, R.K. Thauer, A. Klein, M. Voges and A. Berkessel. 1989. Methyl-coenzyme-M reductase from Methanobacterium thermoautotrophicum. Eur. J. Biochem. 184:63-68.

Hill, J.E., D.A. Scott, S.H. Luo and R. Docampo. 2000. Cloning and functional expression of a gene encoding a vacuolar-type proton-translocating pyrophosphatase from Trypanosoma cruzi. Biochem. J. 351:281-288.

Hippe, H., D. Caspari, K. Fiebig and G. Gottschalk. 1979. Utilization of trimethylamine and other N-methyl compounds for growth and methane formation by Methanosarcina barkeri. Proc. Natl. Acad. Sci. 76:494-498.

Ide, T., S. Bäumer and U. Deppenmeier. 1999. Energy conservation by the $\mathrm{H}_{2}$ :heterodisulfide oxidoreductase from Methanosarcina mazei Gö1: identification of two proton-translocating segments. J. Bacteriol. 181:4076-4080.

Ikeda, M., E. Tanabe, M. Rahman, H. Kadowaki, C. Moritani, R. Akagi, Y. Tanaka, M. Maeshima and Y. Watanabe. 1999. A vacuolar $\mathrm{H}^{+}$-pyrophosphatase in Acetabularia acetabulum: molecular cloning and comparison with higher plants and a bacterium. J. Exp. Bot. 50:139-140.

Keltjens, J.T., R. Van Erp, R.J. Mooijaart, C. Van der Drift and G.D. Vogels. 1988. Inorganic pyrophosphate synthesis during methanogenesis from methylcoenzyme $\mathrm{M}$ by cell free extracts of Methanobacterium thermoautotrophicum. Eur. J. Biochem. 172:471-476.

Klenk, H.P., R.A. Clayton, J.F. Tomb, et al. 1997. The complete genome sequence of the hyperthermophilic, sulphate-reducing archaeon Archaeoglobus fulgidus. Nature 390:364-370.

Luo, S., N. Marchesini, S.N.J. Moreno and R. Docampo. 1999. A plant-like vacuolar $\mathrm{H}^{+}$-pyrophosphatase in Plasmodium falciparum. FEBS Lett. 460:217-220.

Maeshima, M. 2000. Vacuolar $\mathrm{H}^{+}$-pyrophosphatase. Biochim. Biophys. Acta 1465:37-51.

Maeshima, M. and S. Yoshida. 1989. Purification and properties of vacuolar membrane proton-translocating inorganic pyrophosphatase from mung bean. J. Biol. Chem. 264:20,068-20,073.

Müller, V., C. Ruppert and T. Lemker. 1999. Structure and function of the $\mathrm{A}_{1} \mathrm{~A}_{0}$-ATPase from methanogenic archaea. J. Bioenerg. Biomemb. 31:15-27.
Nakanishi, Y., T. Saijo, Y. Wada and M. Maeshima. 2001. Mutagenic analysis of functional residues in putative substrate-binding site and acidic domains of vacuolar $\mathrm{H}^{+}$-pyrophosphatase. J. Biol. Chem. 276:7654-7660.

Nelson, K.E., R.A. Clayton, S.R. Gill, et al. 1999. Evidence for lateral gene transfer between Archaea and Bacteria from genome sequence of Thermotoga maritima. Nature 399: 323-329.

Peinemann, S. 1989. Kopplung von ATP-Synthese und Methanogenese in Vesikelpräparationen des methanogenen Bakteriums Stamm Gö1. Ph.D. Thesis, Univ. Göttingen, pp 33-34.

Rea, P.A. and R.J. Poole. 1993. Vacuolar $\mathrm{H}^{+}$-translocating pyrophosphatase. Annu. Rev. Plant Physiol. Plant Mol. Biol. 44:157-180.

Rea, P.A. and D. Sanders. 1987. Tonoplast energization: Two $\mathrm{H}^{+}$ pumps, one membrane. Physiol. Plant. 71:131-141.

Rea, P.A., Y. Kim, V. Sarafian, R.J. Poole, J.M. Davies and D. Sander. 1992. Vacuolar $\mathrm{H}^{+}$-translocating pyrophosphatases: a new category of ion translocase. Trends Biochem. Sci. 17:348-353.

Redenbach, M., H.M. Kieser, D. Denapaite, A. Eichner, J. Cullum, H. Kinashi and D.A. Hopwood. 1996. A set of ordered cosmids and a detailed genetic and physical map for the $8 \mathrm{Mb}$ Streptomyces coelicolor A3(2) chromosome. Mol. Microbiol. 21:77-96.

Saheki, S., A. Takeda and T. Shimazu. 1985. Assay of inorganic phosphate in the mild $\mathrm{pH}$ range, suitable for measurement of glycogen phosphorylase activity. Anal. Biochem. 148:277-281.

Schöcke, L. and B. Schink. 1998. Membrane-bound proton-translocating pyrophosphatase of Syntrophus gentianae, a syntrophically benzoate-degrading fermenting bacterium. Eur. J. Biochem. 256:589-594.

Silvula, T., A. Salminen, A.N. Parfenyev, P. Pohjanjoki, A. Goldman, B.S. Cooperman, A.A. Baykov and R. Lahti. 1999. Evolutionary aspects of inorganic pyrophosphatase. FEBS Lett. 454:75-80.

Smith, D.R., L.A. Doucette-Stamm, C. Deloughery, et al. 1997. Complete genome sequence of Methanobacterium thermoautotrophicum $\Delta \mathrm{H}$ : Functional analysis and comparative genomics. J. Bacteriol. 179:7135-7155.

Takasu, A., Y. Nakanishi, T. Yamauchi and M. Maeshima. 1997. Analysis of substrate binding site and carboxyl terminal region of vacuolar $\mathrm{H}^{+}$-pyrophosphatase of mung bean with peptide antibodies. J. Biochem. 122:883-889.

Yang, S.J., S.S. Jiang, S.Y. Kuo, S.H. Hung, M.F. Tam and R.L. Pan. 1999. Localization of a carboxylic residue possibly involved in the inhibition of vacuolar $\mathrm{H}^{+}$-pyrophosphatase by $N, N$-dicyclohexylcarbodiimide. Biochem. J. 342:641-646.

Zhen, R.G., E.J. Kim and P.A. Rea. 1997. Acidic residues necessary for pyrophosphate-energized pumping and inhibition of the vacuolar $\mathrm{H}^{+}$-pyrophosphatase by $N, N^{\prime}$-dicyclohexylcarbodiimide. J. Biol. Chem. 272:22,340-22,348. 

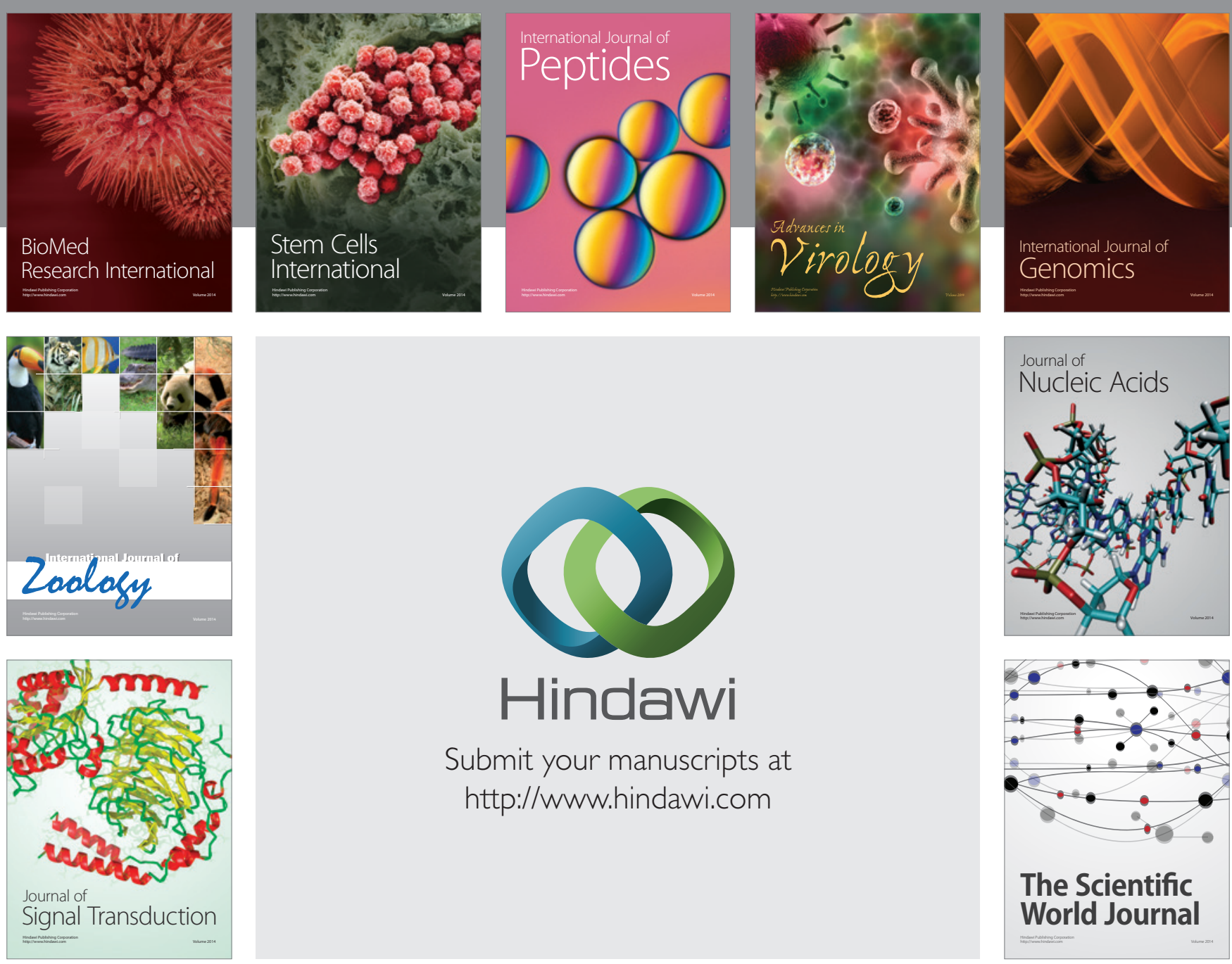

Submit your manuscripts at

http://www.hindawi.com
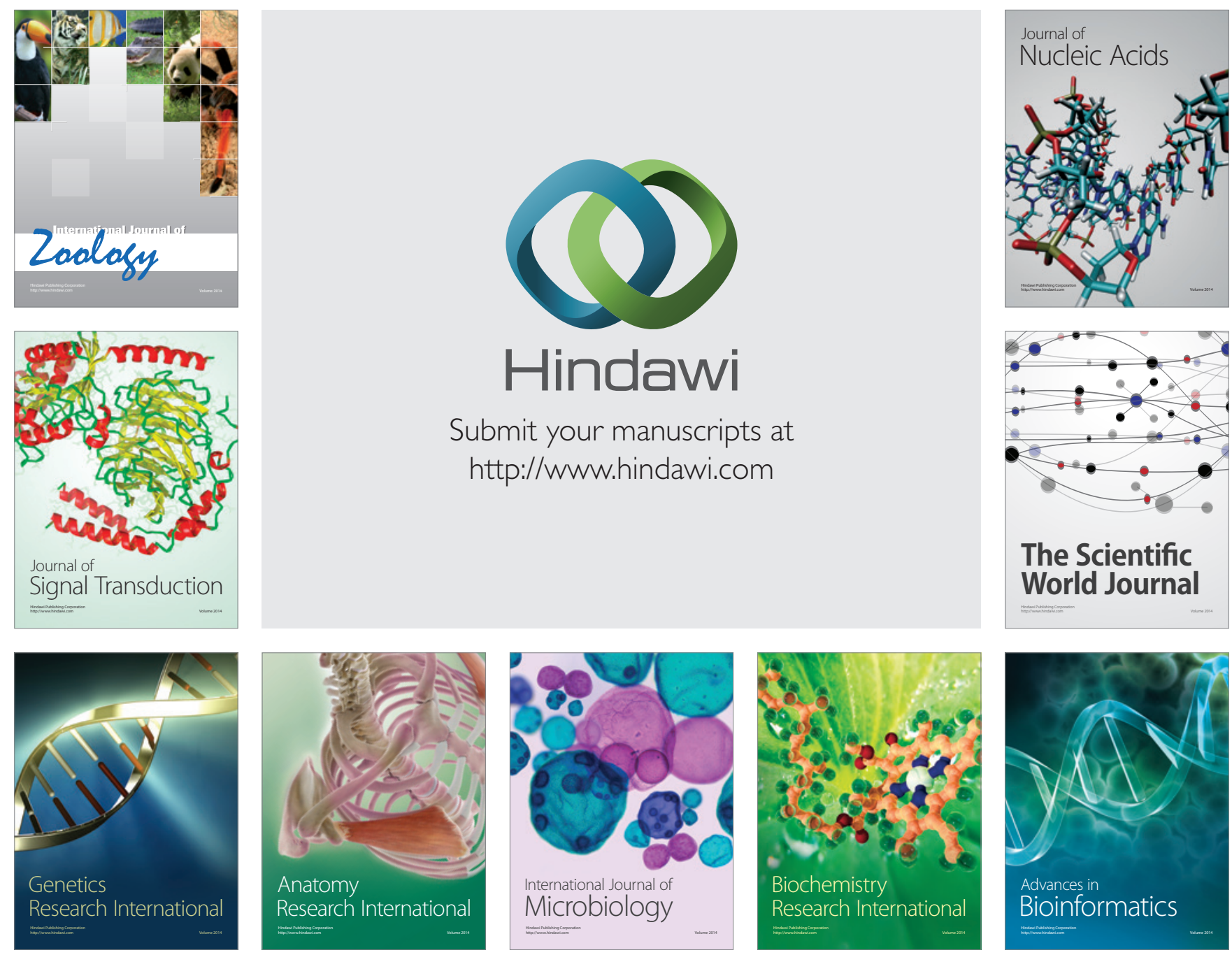

The Scientific World Journal
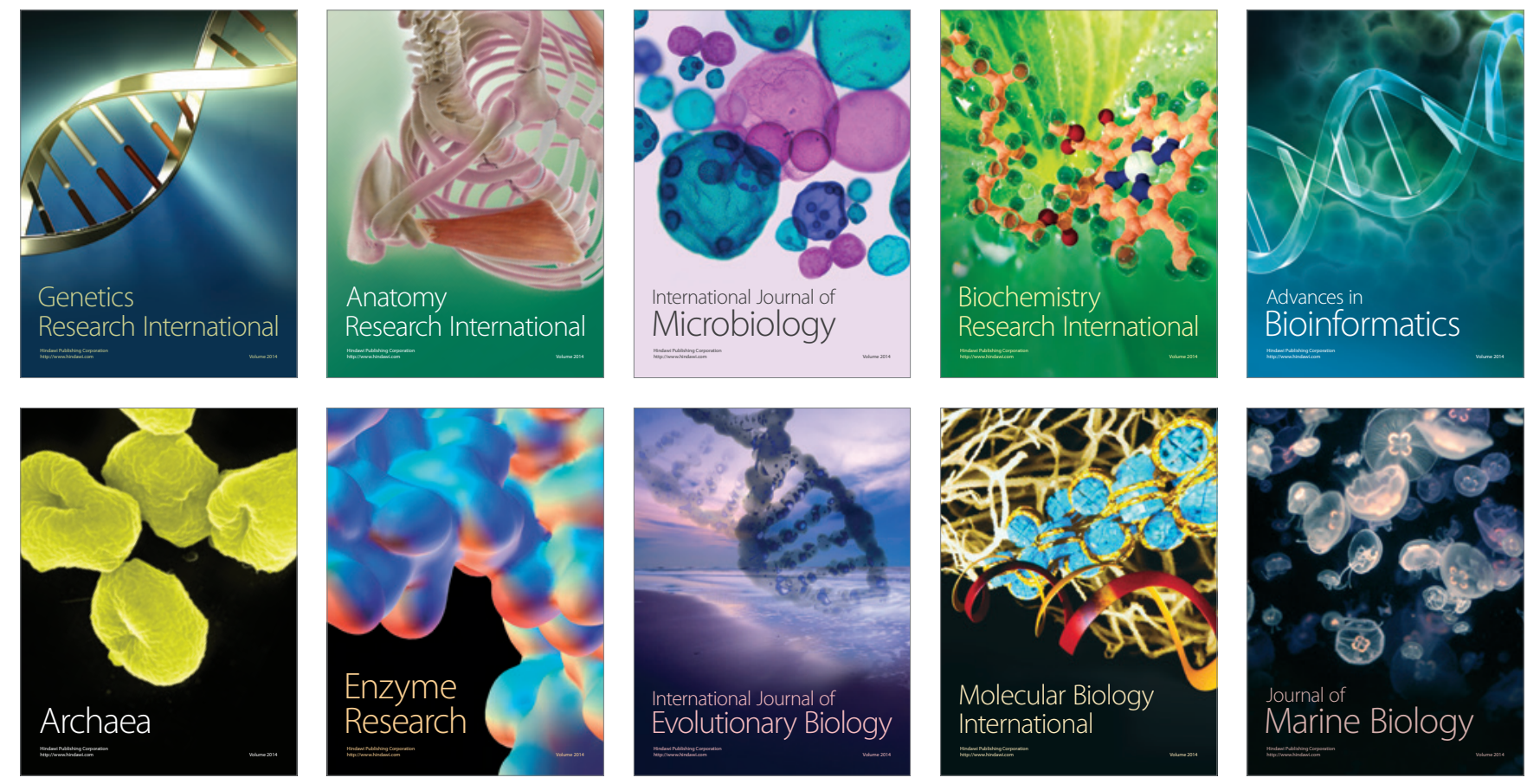